\title{
Recurrence and transience of diffusions in a half-space
}

\author{
SRINIVASAN BALAJI and SUNDARESWARAN RAMASUBRAMANIAN* \\ Indian Statistical Institute, Bangalore Centre, 8th Mile, Mysore Road, R. V. College Post, \\ Bangalore 560 059, India
}

For non-degenerate diffusions in the half-space with oblique reflection, a dichotomy between recurrence and transience is established; convenient characterizations of recurrence and transience are given. Verifiable criteria for recurrence/transience are derived in terms of the generator and the boundary operator. Using these criteria, 'real variables proofs' of some results due to Rogers, concerning reflecting Brownian motion in a half-plane, are obtained. The problem of transience down a side in the case of diffusions in the half-plane is dealt with. Positive recurrence of diffusions in halfspace is also considered; it is shown that the hitting time of any open set has finite expectation if there is just one positive recurrent point.

Keywords: boundary operator; generator; Lyapunov function; oblique reflection; positive recurrence; recurrence; reflecting diffusions; stopping time; strong Feller property; strong Markov property; transience

\section{Introduction}

Recurrence and transience of diffusion processes in $\mathbb{R}^{d}$ have been studied by several authors (see Khasminskii 1960; Friedman 1975; Bhattacharya 1978; Pinsky 1987; Ichihara 1978); these authors give verifiable conditions on the diffusion coefficients (or on the generator) for recurrence/transience. One might consult Pinsky (1995) for an up-to-date review of the known methods and results for the recurrence classification for diffusion processes without reflection. (For corresponding recurrence classification results on Markov chains using martingale ideas based on stochastic analogues of Lyapunov functions, see Meyn and Tweedie (1993a; 1993b) and the references given therein.)

In this paper, we study recurrence, transience and positive recurrence of non-degenerate diffusion processes in the half-space/half-plane, with oblique reflecting boundary conditions at the boundary.

If the state space is a bounded smooth domain, then the reflecting diffusion, being a Feller continuous strong Markov process on a compact space, has an invariant probability measure and hence is positive recurrent (see Bhattacharya and Waymire 1990, p. 230). Therefore problems of interest would be in unbounded domains, like the half-space.

${ }^{*}$ To whom correspondence should be addressed. 
Another case which can easily be dealt with is the class of diffusions in the half-space $\left\{x_{1}>0\right\}$ with conormal reflection at the boundary. Let $L$ denote the generator. The coefficients can be extended in a canonical fashion to $\mathbb{R}^{d}$; and we shall denote once again by $L$ the generator of the corresponding diffusion in $\mathbb{R}^{d}$. If $\left\{U(t)=\left(U_{1}(t), U_{2}(t), \ldots\right.\right.$, $\left.\left.U_{d}(t)\right): t \geqslant 0\right\}$ is the $L$-diffusion in $\mathbb{R}^{d}$, then $\left\{\tilde{U}(t)=\left(\left|U_{1}(t)\right|, U_{2}(t), \ldots, U_{d}(t)\right): t \geqslant 0\right\}$ is the diffusion in the half-space with conormal reflection at the boundary (see Bhattacharya and Waymire 1990). Clearly $\tilde{U}$ is recurrent if and only if $U$ is.

We now outline briefly the organization of the paper. In Section 2 we establish the dichotomy between recurrence and transience (starting, of course, with the natural definitions), and derive criteria for recurrence and transience. We give proofs only when they differ from the case of diffusions (in $\mathbb{R}^{d}$ ) without boundary conditions. (See case (ii) in the proof of Lemma 2.2(a) and the proof of (c) $\Rightarrow$ (d) in Proposition 2.3.) The main difference is the following. It is not clear if an analogue of Lemma 2.3(b) of Bhattacharya (1978) holds in the case of reflecting diffusions. (Of course, maximum principles under stronger differentiability conditions are available as in Protter and Weinberger (1967).) Applying the results of Section 2, in Section 3 we study the case of Brownian motion in the upper half-plane with variable skew reflection; this leads to a real variables proof of some results of Rogers (1991) concerning the same problem (Rogers had used complex analytic tools, in particular the so-called Pick functions).

In Section 4 we consider the diffusion $\left\{\left(X_{1}(t), X_{2}(t)\right)\right\}$ in the space $\left\{x_{1}>0\right\}$ with $\left\{X_{1}(t)\right\}$ being recurrent; we give conditions for $\left\{X_{2}(t)\right\}$ to go to $-\infty$ a.s. Positive recurrence is considered in Section 5; it is shown that if one point is positive recurrent then the hitting time of any open set has finite expectation, and in particular the diffusion itself is positive recurrent. Miscellaneous examples and comments are given in Section 6.

\section{Criteria for recurrence and transience}

Let $D=\left\{x \in \mathbb{R}^{d}: x_{1}>0\right\}, d \geqslant 2$, and $\bar{D}=D \cup \partial D$. We have the coefficients $a, b$ defined on $\bar{D}, \gamma$ defined on $\partial D$ satisfying the following conditions:

(A1) For each $x \in \bar{D}, a(x)=\left(\left(a_{i j}(x)\right)\right)_{1 \leqslant i, j \leqslant d}$ is a $d \times d$ real symmetric positive definite matrix; there exist $\lambda_{1}, \lambda_{2}>0$ such that, for any $x \in \bar{D}$, any eigenvalue of $a(x) \in\left[\lambda_{1}, \lambda_{2}\right]$; and $a_{i j}(\cdot)$ are bounded and Lipschitz continuous.

(A2) For each $x \in \bar{D}, b(x)=\left(b_{1}(x), b_{2}(x), \ldots, b_{d}(x)\right)$ is a vector in $\mathbb{R}^{d} ; b_{i}(\cdot)$ are bounded Lipschitz continuous.

(A3) For each $x \in \partial D, \gamma(x)=\left(1, \gamma_{2}(x), \gamma_{3}(x), \ldots, \gamma_{d}(x)\right)$ is a vector in $\mathbb{R}^{d}$, and each $\gamma_{i} \in C_{b}^{3}(\partial D)$.

Let the generator $L$ and the boundary operator $J$ be given by

$$
L f(x)=\frac{1}{2} \sum_{i, j=1}^{d} a_{i j}(x) \frac{\partial^{2} f(x)}{\partial x_{i} \partial x_{j}}+\sum_{i=1}^{d} b_{i}(x) \frac{\partial f(x)}{\partial x_{i}}, \quad x \in D,
$$




$$
J f(x)=\frac{\partial f(x)}{\partial x_{1}}+\sum_{i=2}^{d} \gamma_{i}(x) \frac{\partial f(x)}{\partial x_{i}}, \quad x \in \partial D .
$$

Let $\Omega=C\left([0, \infty)\right.$ : $\left.\mathbb{R}^{d}\right)$ be endowed with the topology of uniform convergence on compacts. Let $X(t)$ denote the $t$ th coordinate map on $\Omega$, that is $X(t, \omega)=: \omega(t)$; let $\left\{\mathscr{B}_{t}\right\}$ be the natural filtration.

Let $\left\{P_{x}: x \in \bar{D}\right\}$ be the $(L, J)$-diffusion on $\bar{D}$, that is,

(i) $P_{x}\{\omega: X(0, \omega)=x, X(t, \omega) \in \bar{D}, \forall t \geqslant 0\}=1$;

(ii) for each $f \in C_{b}^{2}\left(\mathbb{R}^{d}\right)$ with $J f \geqslant 0$ on $\partial D$,

$$
f(X(t))-\int_{0}^{t} 1_{D}(X(s)) L f(X(s)) \mathrm{d} s \text { is a } P_{x} \text {-submartingale. }
$$

Moreover, there exists a continuous, non-decreasing, progressively measurable process $\xi(t)$ on $\Omega$ such that

(i) $\xi(t)=\int_{0}^{t} 1_{\partial D}(X(s)) \mathrm{d} \xi(s)$;

(ii) for each $f \in C_{\mathrm{b}}^{2}\left(\mathbb{R}^{d}\right)$,

$$
f(X(t))-\int_{0}^{t} 1_{D}(X(s)) L f(X(s)) \mathrm{d} s-\int_{0}^{t} 1_{\partial D}(X(s)) J f(X(s)) \mathrm{d} \xi(s) \text { is a } P_{x} \text {-martingale. }
$$

The $(L, J)$-diffusion $\left\{P_{x}: x \in \bar{D}\right\}$ is strong Markov and Feller continuous; or equivalently under $\left\{P_{x}\right\}$ the process $\{X(t): t \geqslant 0\}$ is strong Markov and Feller continuous. By the existence of a continuous transition density under the conditions (A1)-(A3), the strong Feller property follows: see Ramasubramanian (1996).

For any open set $V$ in $\bar{D}$, define the stopping time

$$
\tau_{V}=\inf \{t \geqslant 0: X(t) \notin V\} \text {. }
$$

Note that we are not assuming $V$ to be bounded. If $V$ is bounded, by Lemma 3 in Ramasubramanian (1986), we have $P_{x}\left(\tau_{V}<\infty\right)=1$ for all $x \in \bar{V}$.

Lemma 2.1. Let $V$ be a bounded open set in $\bar{D}, g$ a bounded measurable function such that, for $x \in V$

$$
g(x)=\mathrm{E}_{x}\left[g\left(X\left(\tau_{V}\right)\right)\right]
$$

Then $g$ is a continuous function on $V$.

Proof. In view of strong Markov, strong Feller properties of $(L, J)$-diffusions, Theorem 13.1 of Dynkin (1965) and Lemma 2.2 of Bhattacharya (1978), it is enough to show that

$$
\lim _{t \downarrow 0} \sup _{x \in \mathrm{K}} P_{x}(|X(t)-x|>\varepsilon)=0,
$$

for any $K \subseteq \bar{D}, K$ compact and $\varepsilon>0$. But this follows from the uniform estimate given in Stroock and Varadhan (1971, p. 181). 
Lemma 2.2 (a) Let $U_{1}, U_{2}$ be open sets in $\bar{D}$ such that $U_{I}$ is non-empty and $\bar{U}_{1} \cap \bar{U}_{2}=\phi$. Let $\sigma_{i}=\inf \left\{t \geqslant 0: X(t) \notin{\overline{\left(U_{i}\right)}}^{c}\right\}, i=1,2$. Then $x \mapsto P_{x}\left(\sigma_{1}<\sigma_{2}\right)$ is a strictly positive continuous function on $\overline{\left(U_{1}\right)^{\mathrm{c}}} \cap \overline{\left(U_{2}\right)^{\mathrm{c}}}$.

(b) Let $U$ be an open set in $\bar{D}$. Then $x \mapsto P_{x}\left(\tau_{U}<\infty\right)$ is a strictly positive continuous function on $U$.

Proof. (a) Let $g(x)=P_{x}\left(\sigma_{1}<\sigma_{2}\right)$, and $x \in \bar{U}_{1}^{\mathrm{c}} \cap \bar{U}_{2}^{\mathrm{c}}$ be arbitrary. Let $V$ be a neighbourhood of $x$ such that $x \in V \subset \bar{V} \subset \overline{\left(U_{1}\right)^{\mathrm{c}}} \cap \overline{\left(U_{2}\right)^{\mathrm{c}}}$. Then we have

$$
g(x)=\mathrm{E}_{x}\left[\mathrm{E}_{X\left(\tau_{V}\right)}\left(1_{\left\{\sigma_{1}<\sigma_{2}\right\}}\right]=\mathrm{E}_{x}\left[g\left(X\left(\tau_{V}\right)\right)\right] .\right.
$$

Hence by Lemma $2.1, g$ is continuous on $V$. It remains to show that $g$ is strictly positive.

Case (i): $x \in D \cap \bar{U}_{1}^{\mathrm{c}} \cap \bar{U}_{2}^{\mathrm{c}}$. Since $(L, J)$ diffusion behaves like $L$-diffusion till hitting $\partial D$, by the support theorem of Stroock and Varadhan (1972), it follows that $g$ is strictly positive.

Case (ii): $x \in \partial D \cap \bar{U}_{1}^{\mathrm{c}} \cap \bar{U}_{2}^{\mathrm{c}}$. Let $\delta>0$ be such that $\overline{B(x: \delta)} \cap \bar{U}_{i}=\phi, i=1,2$. Then by the strong Markov property, $g(x)=\mathrm{E}_{x}\left[g\left(X\left(\tau_{B}\right)\right)\right]$, where $\tau_{B}=\inf \{t \geqslant 0: X(t) \notin B(x: \delta)\}$. Suppose $g(x)=0$. Then $P_{x}\left(\sigma_{1}<\sigma_{2}\right)=0, P_{x} X\left(\tau_{B}\right)^{-1}$-a.s. Since $(L, J)$-diffusion does not hit $\partial D \cap \partial B(x: \delta)$, which is a $(d-2)$-dimensional manifold (see Theorem 3.7 of Ramasubramanian 1988), it follows that $P_{z}\left(\sigma_{1}<\sigma_{2}\right)=0$ for some $z \in D \cap \partial B(x: \delta)$. This contradicts case (i). Hence $g$ is strictly positive.

(b) Follows directly from (a) by taking $U_{1}=\operatorname{Int}\left(U^{\mathrm{c}}\right)$ and $U_{2}=\phi$.

Definition. (a) A point $x \in \bar{D}$ is said to be a recurrent point for $(L, J)$-diffusion if, for every $\varepsilon>0$,

$$
P_{x}(X(t) \in B(x: \varepsilon) \text { for a sequence of } t \text { 's } \uparrow \infty)=1 .
$$

(b) A point $x \in \bar{D}$ is a transient point for the $(L, J)$-diffusion if

$$
P_{x}\left(\lim _{t \rightarrow \infty}|X(t)|=\infty\right)=1 .
$$

If all the points are recurrent (transient) then the diffusion is recurrent (transient).

Proposition 2.3. Assume (A1)-(A3). The following statements are equivalent.

(a) $x_{0} \in \bar{D}$ is a recurrent point.

(b) $P_{x_{0}}(X(t) \in U$ for some $t \geqslant 0)=1$, for all non-empty open sets $U \subset \bar{D}$.

(c) There exist $z_{0} \in \bar{D}, 0<r_{0}<r_{1}, y \in \partial B\left(z_{0}: r_{1}\right)$ such that $P_{y}(\tau<\infty)=1$, where $\tau=\inf \left\{t \geqslant 0: X(t) \in \overline{\left.B\left(z_{0}: r_{0}\right)\right\}}\right.$.

(d) There exists a compact set $K \subset \bar{D}$ such that $P_{x}(X(t) \in K$ for some $t \geqslant 0)=1$, for all $x \in \bar{D}$.

(e) $P_{x}(X(t) \in U$ for some $t \geqslant 0)=1$, for all $x \in \bar{D}$ and for all non-empty open sets $U \subset \bar{D}$.

(f) $P_{x}(X(t) \in U$ for a sequence of $t$ 's $\uparrow \infty)=1$, for all $x \in \bar{D}$ and for all non-empty open sets $U \subset \bar{D}$.

(g) (L, J)-diffusion is recurrent. 
Proof. We will prove only (a) $\Rightarrow$ (b) and (c) $\Rightarrow$ (d); proofs of other implications are either trivial or analogous to the corresponding implications in Bhattacharya (1978).

(a) $\Rightarrow$ (b). Let $x_{0} \in \bar{D}$ be a recurrent point, and assume, without loss of generality, that $x_{0} \notin U$. Let $B$ be a ball such that $\bar{B} \subset U$. Choose $\varepsilon>0$ such that $\overline{B\left(x_{0}, \varepsilon\right)} \cap \bar{B}=\phi$. Let $U_{1}$ be a bounded open set such that $\overline{B\left(x_{0}, \varepsilon\right)} \cup \bar{B} \subset U_{1}$. By Lemma 2.2, and as the diffusion exits out of bounded sets in finite time, we have

$$
\inf _{y \in \partial U_{1}} P_{y}\left(\sigma_{1}<\sigma_{2}\right)>0 \text {, }
$$

where $\sigma_{1}=\tau_{\bar{B}^{c}}$ and $\sigma_{2}=\tau_{\overline{B\left(x_{0}, \varepsilon\right)^{c}}}$. The rest of the proof follows as proof of (a) $\Rightarrow(\mathrm{b})$ in Proposition 3.1 of Bhattacharya (1978).

(c) $\Rightarrow(\mathrm{d})$. Let $K=\overline{B\left(z_{0}: r_{0}\right)} ; y \in \partial B\left(z_{0}: r_{1}\right)$. By (c) we have $P_{y}(\tau<\infty)=1$.

Case (i): $y \in D$. Define

$$
V(x)=1-P_{x}(\tau<\infty) .
$$

By Lemma 2.2, $V$ is continuous on $K^{\mathfrak{c}}$. By the strong Markov property

$$
0=V(y)=\mathrm{E}_{y}[V(X(\eta))],
$$

where $\eta$ is the exit time from $B(y: \delta)$ with $\overline{B(y: \delta)} \cap K=\phi, B(y: \delta) \subset D$. By (2.12) we have $V(z)=0, P_{y} X(\eta)^{-1}$-a.s. Now by the support theorem for $L$-diffusions (see Stroock and Varadhan 1972), and continuity of $V, V(z)=0$ for all $z \in \partial B(y: \delta)$. This holds for all sufficiently small $\delta<\left(r_{1}-r_{0}\right) \wedge d(y, \partial D)$. If $z \in D \cap K^{\mathrm{c}}$, then one can find points $y_{0}, y_{1}, \ldots, y_{k+1} \in D \cap K^{\mathrm{c}}$ such that $y_{0}=y,\left|y_{j+1}-y_{j}\right|<\left(\left|y_{j}-z_{0}\right|-r_{0}\right) \wedge d\left(y_{j}, \partial D\right)$ and $y_{k+1}=z$. By repeating the above argument, we find

$$
V\left(y_{0}\right)=V\left(y_{1}\right)=\ldots=V(z)=0 .
$$

Thus $V \equiv 0$ on $K^{\mathrm{c}} \cap D$. By continuity, $V \equiv 0$ on $K^{\mathrm{c}}$ and hence on $\bar{D}$.

Case (ii): $y \in \partial D$. As in equation (2.12) we have $0=V(y)=E_{y}[V(X(\eta))]$ by the strong Markov property. Hence $V(X(\eta))=0, P_{y}$ a.s. Since $(L, J)$-diffusion does not hit $\partial D \cap \partial B(y: \delta)$ (see Ramasubramanian 1988) we have $V(z)=0$ for some $z \in D \cap K^{\mathrm{c}}$. Thus the problem is reduced to case (i). Hence the proposition is proved.

For any compact set $K$ define $\sigma_{K}=\inf \left\{t \geqslant 0: X_{t} \in K\right\}$.

Theorem 2.4. Assume (A1)-(A3).

(a) (Dichotomy.) (L, J)-diffusion is not recurrent if and only if $(L, J)$-diffusion is transient.

(b) $(L, J)$-diffusion is recurrent if and only if there exist a compact set $K$ with non-empty interior, a point $x \in K^{\mathrm{c}}$ and a measurable function $u$ such that

(i) $u(z) \uparrow \infty$ as $|z| \uparrow \infty$;

(ii) $\mathrm{E}_{x}\left[u\left(X\left(\sigma_{K}\right)\right)\right] \leqslant u(x)$.

(c) $(L, J)$-diffusion is transient if and only if there exist a compact set $H$ with non-empty interior, $y \in H^{\mathrm{c}}$ and a measurable function $u$ such that 
(i) $\mathrm{E}_{y}\left[1_{\left\{\sigma_{H}<\infty\right\}} u\left(X\left(\sigma_{H}\right)\right)\right] \leqslant u(y)$;

(ii) $u(y)<\inf _{z \in H} u(z)$.

Proof. (a) If $(L, J)$-diffusion is transient then it trivially follows that $(L, J)$-diffusion is not recurrent. Now let us suppose that $(L, J)$-diffusion is not recurrent. Let $x \in \bar{D}$ be arbitrary and choose $r_{0}, r_{1}$ such that $|x|<r_{0}<r_{1}$. Put $\delta_{1}=\sup _{|y|=r_{1}} P_{y}\left(\sigma_{0}<\infty\right)$, where $\sigma_{0}=\inf \left\{t \geqslant 0: X(t) \notin \overline{B\left(0: r_{0}\right)^{c}}\right\}$. Since no point in $\bar{D}$ is recurrent by the previous proposition, we have $P_{y}\left(\sigma_{0}<\infty\right)<1$ for all $y$ such that $|y|=r_{1}$. Now as $y \mapsto P_{y}\left(\sigma_{0}<\infty\right)$ is a continuous function, we have $\delta_{1}<1$. Hence, proceeding as in the proof of Theorem 3.2(b) of Bhattacharya (1978), we get the result.

(b) Necessity. Let $u$ be a function such that $u(z)=\hat{u}(|z|)$, where $\hat{u}$ is a strictly increasing function with $\lim _{r \rightarrow \infty} \hat{u}(r)=\infty$. Let $K=\overline{B(0: 1)}$ and choose $x$ such that $|x|>1$. As the diffusion is recurrent, $\left|X\left(\sigma_{K}\right)\right|=1, P_{x}$ a.s. Hence we have

$$
\mathrm{E}_{x}\left[u\left(X\left(\sigma_{K}\right)\right)\right]=\hat{u}(1)<u(x) .
$$

Sufficiency. Suppose the diffusion is not recurrent and so by part (a) it is transient. Let $A=\left\{\sigma_{K}<\infty\right\}$. By transience we see that $P_{x}\left(A^{\mathrm{c}}\right)>0$; and again by transience and (b)(i) note that $u\left(X\left(\sigma_{K}\right)\right)=\infty$ on $A^{\mathrm{c}}$. Hence we have $\mathrm{E}_{x}\left[u\left(X\left(\sigma_{K}\right)\right)\right]=\infty$, which is a contradiction.

(c) Necessity. Let $\mathrm{H}=\overline{B(0: 1)}$. Put $u(x)=P_{x}\left(\sigma_{H}<\infty\right), x \in \bar{D}$. Then we have $u(x)=\mathrm{E}_{x}\left[1_{\left\{\sigma_{H}<\infty\right\}} 1\right]=\mathrm{E}_{x}\left[1_{\left\{\sigma_{H}<\infty\right\}} u\left(X\left(\sigma_{H}\right)\right)\right]$, since $u\left(X\left(\sigma_{H}\right)\right)=1$ on $\left\{\sigma_{H}<\infty\right\}$. By transience $u(x)<1$ for $|x|>1$, but $u(z)=1$ for all $z \in H$. Hence (ii) is also satisfied.

Sufficiency. Suppose the diffusion is not transient. Hence by part (a) it is recurrent. Therefore by (i) and (ii) of (c),

$$
\begin{aligned}
u(y) & \geqslant \mathrm{E}_{y}\left[1_{\left\{\sigma_{H}<\infty\right\}} u\left(X\left(\sigma_{H}\right)\right)\right]=\mathrm{E}_{y}\left[u\left(X\left(\sigma_{H}\right)\right)\right] \\
& \geqslant \inf _{z \in H} u(z)>u(y),
\end{aligned}
$$

and hence we have a contradiction. Therefore the diffusion is transient.

We now derive some corollaries which are analogues of Proposition 3.1 and 3.2 of Pinsky (1987).

Corollary 2.5. If there exist $r_{0}>0$ and $u \in C^{2}\left(\mathbb{R}^{d} \backslash B\left(0: r_{0} / 2\right)\right)$ such that
(i) $u(x) \uparrow \infty$ as $|x| \uparrow \infty$;
(ii) $\operatorname{Lu(x)} \leqslant 0,|x| \geqslant r_{0}$;
(iii) $J u(x) \leqslant 0,\left\{|x| \geqslant r_{0}\right\} \cap \partial D$,

then $(L, J)$-diffusion is recurrent.

Proof. By Itô's lemma, the optional sampling theorem and by conditions (ii), (iii) above, we have

$$
\mathrm{E}_{x}\left[u\left(X\left(t \wedge \sigma_{K}\right)\right)\right] \leqslant u(x)
$$


where $K=\overline{B\left(0: r_{0}\right)}$. Let $A=\left\{\sigma_{\mathrm{K}}<\infty\right\}$. If $P_{x}\left(A^{\mathrm{c}}\right)>0$ then by dichotomy (Theorem 2.4(a)) the diffusion is transient and hence $\lim _{t \rightarrow \infty}|X(t, \omega)|=\infty$, for $\omega \in A^{\mathrm{c}}$. Hence as $u$ can be taken to be non-negative without loss of generality,

$$
\lim _{t \rightarrow \infty} \mathrm{E}_{x}\left[u\left(X\left(t \wedge \sigma_{K}\right)\right)\right] \geqslant \lim _{t \rightarrow \infty} \mathrm{E}_{x}\left[1_{A^{c}} u\left(X\left(t \wedge \sigma_{K}\right)\right)\right]=\infty .
$$

Note that (2.17) contradicts (2.16). Hence $P_{x}\left(A^{\mathrm{c}}\right)=0$.

Now letting $t \rightarrow \infty$ we have

$$
\mathrm{E}_{x}\left[u\left(X\left(\sigma_{K}\right)\right)\right] \leqslant u(x) .
$$

By Theorem 2.4(b) we have that the diffusion is recurrent.

Corollary 2.6. If there exist $r_{0}>0$ and a function $u \in C_{b}^{2}\left(\mathbb{R}^{d} \backslash\left(B\left(0: r_{0} / 2\right)\right)\right.$ such that

(i) $L u(x) \leqslant 0,|x| \geqslant r_{0}$;

(ii) $J u(x) \leqslant 0,\left\{|x| \geqslant r_{0}\right\} \cap \partial D$;

(iii) there is a point $x_{0}$ such that $\left|x_{0}\right|>r_{0}$ and $u\left(x_{0}\right)<\inf _{|x|=r_{0}} u(x)$, then the diffusion is transient.

Proof. Let $K=\overline{B\left(0: r_{0}\right)}$. Without loss of generality let us take $u \geqslant 0$. By Itô's lemma, the optional sampling theorem and by conditions (i), (ii) above, we have

$$
\mathrm{E}_{x_{0}}\left[u\left(X\left(t \wedge \sigma_{K}\right)\right)\right] \leqslant u\left(x_{0}\right) .
$$

Now

$$
\begin{gathered}
\mathrm{E}_{x_{0}}\left[1_{\left\{\sigma_{K}<\infty\right\}} u\left(X\left(\sigma_{K}\right)\right)\right]=\lim _{t \uparrow \infty} \mathrm{E}_{x_{0}}\left[1_{\left\{\sigma_{K} \leqslant t\right\}} u\left(X\left(\sigma_{K}\right)\right)\right] \\
\leqslant \lim _{t \uparrow \infty}\left\{\mathrm{E}_{x_{0}}\left[1_{\left\{\sigma_{K} \leqslant t\right\}} u\left(X\left(\sigma_{K}\right)\right)\right]+\mathrm{E}_{x_{0}}\left[1_{\left\{\sigma_{K}>t\right\}} u(X(t))\right]\right\} \\
=\lim _{t \rightarrow \infty} \mathrm{E}_{x_{0}}\left[u\left(X\left(t \wedge \sigma_{K}\right)\right)\right] \leqslant u\left(x_{0}\right) .
\end{gathered}
$$

Hence by Theorem 2.4(c) we have transience.

Now let us give some criteria for recurrence and transience of diffusions in terms of the coefficients of $L$ and $J$. These are analogues to the criteria in Bhattacharya (1978). Let $L, J$ be defined as in (2.1), (2.2).

Define

$$
\begin{aligned}
& A(x)=\sum_{i, j=1}^{d} a_{i j}(x) \frac{x_{i} x_{j}}{|x|^{2}}, \\
& B(x)=\sum_{i=1}^{d} a_{i i}(x), \\
& C(x)=2 \sum_{j=1}^{d} x_{i} b_{i}(x) .
\end{aligned}
$$


Put

$$
\begin{aligned}
& \bar{\beta}(r)=\sup _{|x|=r} \frac{B(x)-A(x)+C(x)}{A(x)} \\
& \underline{\beta}(r)=\inf _{|x|=r} \frac{B(x)-A(x)+C(x)}{A(x)} \\
& \bar{I}(r)=\int_{1}^{r} \frac{\bar{\beta}(u)}{u} \mathrm{~d} u ; \underline{I}(r)=\int_{1}^{r} \frac{\underline{\beta}(u) \mathrm{d} u}{u} .
\end{aligned}
$$

Proposition 2.7. Assume (A1)-(A3).

(a) Let $u(x)=\int_{1}^{|x|} \exp (-\bar{I}(r)) \mathrm{d} r$. If $u(x) \rightarrow \infty$ as $|x| \rightarrow \infty$ and $J u(x) \leqslant 0$ for $x \in \partial D,|x|>1$, then the diffusion is recurrent.

(b) Let $v(x)=\int_{1}^{|x|} \exp (-\underline{I}(r)) \mathrm{d} r$. If $\lim _{x \rightarrow \infty} v(x)<\infty$ and $J v(x) \geqslant 0$ for $x \in \partial D,|x|>1$, then the diffusion is transient.

Proof. This easily follows from Corollories 2.5 and 2.6 and the proof of Theorem 3.3 in Bhattacharya (1978).

Remark 2.8. Note that $(L, J)$-diffusion can be transformed to $\left(\tilde{L}, \partial / \partial_{x_{1}}\right)$-diffusion through a $C^{2}$-diffeomorphism of $\bar{D}$ (see Ramasubramanian 1986). Let $\tilde{a}, \tilde{b}$ denote the coefficents of $\tilde{L}$. Define $\tilde{A}, \tilde{B}, \tilde{C}$ analogous to $A, B, C$ above with $\tilde{a}, \tilde{b}$ replaced by $a, b$.

Define

$$
\begin{aligned}
& \tilde{\beta}(r)=\sup _{|x|=r} \frac{\tilde{B}(x)-\tilde{A}(x)+\tilde{C}(x)}{\tilde{A}(x)} \\
& \tilde{u}(x)=\int_{1}^{|x|} \exp \left(-\int_{1}^{r} \frac{\tilde{\beta}(u)}{u} \mathrm{~d} u\right) \mathrm{d} r
\end{aligned}
$$

Note that $\partial \tilde{u} / \partial x_{1}=0$ on $\partial D$. Thus if $\tilde{u}(x) \rightarrow \infty$ as $|x| \rightarrow \infty$, we have that $(L, J)$-diffusion is recurrent. Similarly we can also have a condition for transience.

Remark 2.9. The boundedness assumptions in (A1), (A2) can be relaxed to linear growth conditions on $a, b$. Under such conditions the $(L, J)$-diffusion is conservative. As in Lemma 2.5 of Bhattacharya (1978), the strong Feller property can be established. It is now clear that the analysis of this section can be carried through under the relaxed assumptions. We omit the details. 


\section{Reflecting Brownian motion in the upper half-plane with variable skew reflection}

In this section we will deal with recurrence and transience of reflecting Brownian motion (RBM) with variable oblique reflection in the upper half-plane. Rogers (1991) has dealt with this problem but has used complex analytic techniques to get the results. Here we will give a real variables proof of these results, using in particular Corollaries 2.5 and 2.6 above.

In this case it is convenient to deal with the problem in polar coordinates. Therefore we shall describe the set-up in Cartesian coordinates as well as in polar coordinates.

Let

$$
\begin{gathered}
S=\left\{\left(x_{1}, x_{2}\right): x_{2}>0,-\infty<x_{1}<\infty\right\}=\{(r, \theta): r>0, \theta \in(0, \pi)\} \\
\partial_{1} S=\left\{\left(x_{1}, 0\right): x_{1}>0\right\}=\{(r, \theta): r>0, \theta=0\} \\
\partial_{2} S=\left\{\left(x_{1}, 0\right): x_{1}<0\right\}=\{(r, \theta): r>0, \theta=\pi\} \\
\partial S=\partial_{1} S \cup \partial_{2} S \cup\{(0,0)\} ; \bar{S}=S \cup \partial S .
\end{gathered}
$$

Here the generator $L$ is the Laplacian, viz.,

$$
L=\frac{1}{2} \Delta=\frac{1}{2}\left(\frac{\partial^{2}}{\partial x_{1}^{2}}+\frac{\partial^{2}}{\partial x_{2}^{2}}\right)=\frac{1}{2}\left(\frac{\partial^{2}}{\partial r^{2}}+\frac{1}{r} \frac{\partial}{\partial r}+\frac{1}{r^{2}} \frac{\partial^{2}}{\partial \theta^{2}}\right) .
$$

For $x \in \partial S$, let $\gamma(x)$ be the direction of reflection and let $\eta(x)$ be the angle that $\gamma(x)$ makes with the normal at $x$, clockwise direction being taken to be positive.

As in Section 2, we will assume that the normal component of $\gamma$ is bounded away from 0 ; Hence without loss of generality, we may take the normal component to be 1 . So we may write $\gamma(x)=\left(\gamma_{1}(x), 1\right)=(\tan \eta(x), 1)$. As $\gamma$ is bounded, and bounded away from the tangential direction, note that there exists a $\beta>0$ such that

$$
-\pi / 2+\beta \leqslant \eta(x) \leqslant \pi / 2-\beta .
$$

Also, we assume that $\eta(x) \in C_{b}^{3}(\partial S)$.

Now the boundary operator $J$ can be written as

$$
\begin{aligned}
J f(x) & =\gamma_{1}(x) \frac{\partial f(x)}{\partial x_{1}}+\frac{\partial f(x)}{\partial x_{2}} \\
& = \begin{cases}\tan \eta(\cdot) \frac{\partial f(\cdot)}{\partial r}+\frac{1}{r} \frac{\partial f(\cdot)}{\partial \theta}, & \text { on } \partial_{1} S \\
-\tan \eta(\cdot) \frac{\partial f(\cdot)}{\partial r}-\frac{1}{r} \frac{\partial f(\cdot)}{\partial \theta}, & \text { on } \partial_{2} S .\end{cases}
\end{aligned}
$$

For $x \in \partial_{1} S$, note that $\eta(x)=\eta(|x|, 0)$, and for $x \in \partial_{2} S$, we have $\eta(x)=\eta(|x|, \pi)$. We will use this notation in the sequel.

Theorem 3.1. (a) If $\liminf _{r \rightarrow \infty} \eta(r, \pi)+\liminf _{r \rightarrow \infty}(-\eta(r, 0))>0$, then the $R B M$ is recurrent. 
(b) If $\limsup _{r \rightarrow \infty} \eta(r, \pi)+\lim \sup _{r \rightarrow \infty}(-\eta(r, 0))<0$, then the RBM is transient.

(c) If $\eta(r, 0)$ is non-decreasing and $\eta(r, \pi)$ is non-increasing, and if

$$
\lim _{r \rightarrow \infty} \eta(r, 0)=\lim _{r \rightarrow \infty} \eta(r, \pi)
$$

then the RBM is recurrent.

Proof. (a) Let $\liminf _{r \rightarrow \infty}(-\eta(r, 0))=\xi_{1}$ and $\liminf _{r \rightarrow \infty} \eta(r, \pi)=\xi_{2}$. By hypothesis $\xi_{1}+\xi_{2}=\varepsilon>0$. Put $\xi_{1}-\varepsilon / 4=\theta_{1}, \xi_{2}-\varepsilon / 4=\theta_{2}$ and $\alpha=\left(\theta_{1}+\theta_{2}\right) / \pi$. Note that $\theta_{1}+\theta_{2}>0$ and hence $\alpha>0$. Now define the function $u$ on the set $\overline{B(0: 1)^{\mathrm{c}}} \cap \bar{S}$ in terms of polar coordinates as follows:

$$
u(r, \theta)=r^{\alpha} \cos \left(\alpha \theta-\theta_{1}\right) .
$$

Clearly $\frac{1}{2} \Delta u=0$. Note that $-\pi / 2<-\theta_{1} \leqslant \alpha \theta-\theta_{1} \leqslant \theta_{2}<\pi / 2$, for all $\theta \in[0, \pi]$. Hence $\left\{\cos \left(\alpha \theta-\theta_{1}\right): \theta \in[0, \pi]\right\}$ is bounded away from 0 . Therefore $u(r, \theta) \rightarrow \infty$ as $r \rightarrow \infty$ since $\alpha>0$. On $\partial_{1} S$ we have

$$
J u(r, \theta)=\alpha r^{\alpha-1} \cos \theta_{1} \tan \eta(r, 0)+\alpha r^{\alpha-1} \sin \theta_{1} .
$$

But since $\liminf _{r \rightarrow \infty}(-\eta(r, 0))=\xi_{1}$, there exists an $s_{1}$ such that for all $r \geqslant s_{1}$, $\eta(r, 0) \geqslant \xi_{1}-\varepsilon / 4=\theta_{1}$. Hence $\tan \eta(r, 0) \leqslant \tan \left(-\theta_{1}\right)$. Consequently as $\alpha>0$, by (3.3), we have on $\partial_{1} S \cap\left\{r>s_{1}\right\}$

$$
J u(r, 0) \leqslant 0 .
$$

Similarly, on $\partial_{2} S$

$$
J u(r, \pi)=-\alpha r^{\alpha-1} \cos \theta_{2} \tan \eta(r, \pi)+\alpha r^{\alpha-1} \sin \theta_{2} .
$$

But as $\liminf _{r \rightarrow \infty} \eta(r, \pi)=\xi_{2}$, we have, for some $s_{2}>0$, for all $r \geqslant s_{2}, \eta(r, \pi) \geqslant$ $\xi_{2}-\varepsilon / 4=\theta_{2}$. Substituting in (3.5) we see that, on $\partial_{2} S \cap\left\{r \geqslant s_{2}\right\}$

$$
J u(r, \pi) \leqslant 0 .
$$

Hence by (3.4) and (3.6), we have on $\left[\partial_{1} S \cap\left\{r \geqslant s_{0}\right\}\right] \cup\left[\partial_{2} S \cap\left\{r \geqslant s_{0}\right\}\right], J u \leqslant 0$, where $s_{0}=\max \left\{s_{1}, s_{2}\right\}$. Now by Corollary 2.5 the process is recurrent.

(b) Let $\lim \sup _{r \rightarrow \infty}(-\eta(r, 0))=\xi_{1}$ and $\lim _{\sup _{r \rightarrow \infty}} \eta(r, \pi)=\xi_{2}$. By hypothesis $\xi_{1}+\xi_{2}=$ $-\varepsilon<0$. Put $\xi_{1}+\varepsilon / 4=\theta_{1}$ and $\xi_{2}+\varepsilon / 4=\theta_{2}$. Note that $\theta_{1}+\theta_{2}<0$ and let $\alpha=\left(\theta_{1}+\theta_{2}\right) / \pi<0$. Define

$$
u(r, \theta)=r^{\alpha} \cos \left(\alpha \theta-\theta_{1}\right) .
$$

Proceeding as in the proof of part (a) with obvious modifications and using Corollary 2.6, it can be proved that RBM is transient in this case.

(c) Let $\lim _{r \rightarrow \infty} \eta(r, 0)=-\theta_{1}$ and $\lim _{r \rightarrow \infty} \eta(r, \pi)=\theta_{2}$. By assumption $\eta(r, 0) \uparrow-\theta_{1}$ and $\eta(r, \pi) \downarrow \theta_{2}$. Define

$$
u(r, \theta)=\log r+\theta \tan \theta_{1} .
$$

With $u$ defined by (3.8) and using Corollary 2.5, recurrence of RBM in this case can be established along similar lines as in part (a). 
Remark 3.2. Parts (a) and (b) of Theorem 3.1 have been proved by Rogers (1991) using complex analytic methods. It may be mentioned that Rogers obtains other results as well concerning RBM with variable reflection field, using Pick functions from complex analysis; see also Rogers (1990). Since two-dimensional Brownian motion is well behaved under conformal mappings, the complex analytic approach as considered by Rogers is a natural tool to use. Observe that Brownian motion in $\mathbb{R}^{2}$ is a critical case as far as recurrence/transience is concerned; that is Brownian motion in $\mathbb{R}^{2}$ just fails to be transient! (The authors thank Prof. Varadhan for this remark.) This aspect is also manifest in part (b) of Theorem 3.1, in the sense that a mild perturbation by a suitable 'reflection field' is enough to make the process transient. Our 'real variables' approach enables us also to consider other critical cases like Example 6.3.

A particular case of part (c), viz., when $\gamma(x) \equiv$ constant, has been dealt with by Williams (1985). In fact our choice of the function $u$ in the above proofs has been inspired by Varadhan and Williams (1985).

Proposition 3.3. Let $\eta(x)$ be the angle of reflection on the boundary $\partial S$ such that it satisfies $\eta\left(x_{1}\right)=\eta\left(x_{1}+1\right)$, that is, we consider periodic reflecting conditions. Now let

$$
\begin{aligned}
& \gamma_{1}=\inf \left\{\eta\left(x_{1}\right): x_{1} \in[0,1]\right\}, \\
& \gamma_{2}=\sup \left\{\eta\left(x_{1}\right): x_{1} \in[0,1]\right\} .
\end{aligned}
$$

Then if $\gamma_{1}=0$ and $\gamma_{1}<\gamma_{2}$, then the RBM is transient.

Proof. Put $u(r, \theta)=r^{\alpha} \cos (\alpha \theta-\eta)-\theta \tan \gamma_{2}$, where $\alpha=-\gamma_{2} / 2$ and $\eta=\delta_{2} / 2$. Then, along the same lines as in the proof of Theorem 3.1(b), we have transience of the process.

Note. The condition in Proposition 3.3 above is not covered by the inequalities of Theorem 3.1(a)-(b).

Remark 3.4. Consider the generator and boundary operator as follows:

$$
\begin{gathered}
\tilde{L} f(x)=\left(m^{2}+1\right) \frac{\partial^{2} f(x)}{\partial x_{1}^{2}}+2 m \frac{\partial^{2} f(x)}{\partial x_{1} \partial x_{2}}+\frac{\partial^{2} f(x)}{\partial x_{2}^{2}}, \quad x \in D, \\
\tilde{J} f(x)=\frac{\partial f(x)}{\partial x_{2}}, \quad x \in \partial D .
\end{gathered}
$$

where $m$ is a positive constant. That is, we consider diffusion with generator $\tilde{L}$ and normal reflection at the boundary. By a transformation of the upper half-plane as in Ramasubramanian (1988), we see that $(\tilde{L}, \tilde{J})$ diffusion is transformed to $(\Delta, J)$-diffusion, where

$$
J f(x)=m \frac{\partial f(x)}{\partial x_{1}}+\frac{\partial f(x)}{\partial x_{2}}
$$


By Theorem 3.1(c) we see that $(\Delta, J)$-diffusion is recurrent. Hence $(\tilde{L}, \tilde{J})$-diffusion is recurrent. It is interesting to note that Proposition 2.7 does not yield any information concerning the recurrence of $(\tilde{L}, \tilde{J})$-diffusion. This is not altogether very surprising because both Theorem 3.3. of Bhattacharya (1978), and Proposition 2.7 work well when the generator and the boundary operator preserve the class of radial functions.

Our proof of Theorem 3.1(a) and a theorem due to Menshikov and Williams immediately suggest the following result concerning passage-time moments.

Proposition 3.5. Suppose the hypothesis of Theorem 3.1(a) holds. Let a be as in the proof of Theorem 3.1(a). Then there exists a positive constant $c<1$ such that $r>0$,

(i) $\mathrm{E}_{z}\left(\tau_{r}^{p}\right)<\infty$, for $p<\alpha / 2,|z|>r$;

(ii) $\mathrm{E}_{z}\left(\tau_{r}^{p}\right)=\infty$, for $p>\alpha / 2,|z|>r c$, where $\tau_{r}=\inf \{t \geqslant 0:|Z(t)|=r\}$.

Proof. Let $u$ be the function as in the proof of Theorem 3.1(a). Then the proposition follows by applying Theorem 4.1 of Menshikov and Williams (1996) to the function $u$. As $J u \leqslant 0$, the proof of Theorem 4.1 essentially goes through, with minor changes.

\section{Transience down a side in the half-plane}

In this section we revert to the notation of Section 2 . Let

$$
D=\left\{\left(x_{1}, x_{2}\right): x_{1}>0,-\infty<x_{2}<\infty\right\} \text {. }
$$

Let

$$
\begin{gathered}
L f(x)=\sum_{i, j=1}^{2} a_{i j}(x) \frac{\partial^{2} f(x)}{\partial x_{i} \partial x_{j}}+\sum_{i=1}^{2} b_{i}(x) \frac{\partial f(x)}{\partial x_{i}}, \quad x \in D, \\
J f(x)=\frac{\partial f(x)}{\partial x_{1}}+\gamma_{2}(x) \frac{\partial f(x)}{\partial x_{2}}, \quad x \in \partial D .
\end{gathered}
$$

be respectively the generator and boundary operator. Let $\left(X_{1}(t), X_{2}(t)\right)$ denote the $(L, J)$ diffusion on $\bar{D}$. Suppose $\left\{X_{1}(t)\right\}$ is recurrent (that is, for any open set $U$ in $[0, \infty)$ and any $x \in \bar{D}, P_{x}\left(X_{1}(t) \in U\right.$ for a sequence of $t$ 's $\left.\left.\uparrow \infty\right)=1\right)$. We give conditions for $\left\{X_{2}(t)\right\}$ to go to $-\infty$ a.s. Similar conditions can be given for $\left\{X_{2}(t)\right\}$ to go to $+\infty$. In this regard let us prove the following proposition.

Proposition 4.1. Let there be a function $u \in C^{2}\left(\mathbb{R}^{2}\right)$ with the properties

(i) $u \geqslant 0$ and $u(x)=\hat{u}\left(x_{2}\right)$;

(ii) $\hat{u}\left(x_{2}\right)$ decreases as $x_{2} \downarrow-\infty$;

(iii) $\hat{u}\left(x_{2}\right)$ increases to $\infty$ as $x_{2} \uparrow \infty$;

(iv) $L u \leqslant 0$ on $D$ and $J u \leqslant 0$ on $\partial D$. 
Then the diffusion is transient, and furthermore

$$
P_{x}\left(\lim _{t \rightarrow \infty} X_{2}(t)=-\infty\right)=1, \quad \forall x \in \bar{D} .
$$

Proof. Let $r \in \mathbb{R}$ be arbitrary but fixed. Let

$$
\begin{aligned}
& \tau_{r}=\inf \left\{t \geqslant 0: X_{2}(t)=r\right\}, \\
& S_{1}=\left\{\left(x_{1}, x_{2}\right): x_{2}>r\right\}, \\
& S_{2}=\left\{\left(x_{1}, x_{2}\right): x_{2}<r\right\} .
\end{aligned}
$$

Step 1. We will show that

$$
\sup _{x \in L} P_{x}\left(\tau_{r}<\infty\right)<1,
$$

for any horizontal line $L \subseteq S_{2}$. This, in particular, implies that the process is transient.

Suppose (4.3) does not hold; then $\sup _{x \in L} P_{x}\left(\tau_{r}<\infty\right)=1$. So given $\varepsilon>0$, there exists an $x^{(0)} \in L$ such that

$$
\begin{gathered}
P_{x^{(0)}}\left(\tau_{r}<\infty\right)>1-\varepsilon / 2, \\
u\left(x^{(0)}\right) \geqslant \mathrm{E}_{x^{(0)}}\left[u\left(X\left(t \wedge \tau_{r}\right)\right)\right] \\
=\mathrm{E}_{x^{(0)}}\left[1_{\left\{\tau_{r} \leqslant t\right\}} u\left(X\left(\tau_{r}\right)\right)\right]+\mathrm{E}_{x^{(0)}}\left[1_{\left\{\tau_{r}>t\right\}} u(X(t))\right] .
\end{gathered}
$$

Let $A=\left\{\tau_{r}<\infty\right\}$ and $A_{T}=\left\{\tau_{r} \leqslant T\right)$. Choose $T$ such that $P_{x^{0}}\left(A_{T}\right)>1-\varepsilon$. This is possible as $A_{T} \uparrow A$. Consequently as $u \geqslant 0$, we get

$$
\begin{aligned}
u\left(x^{(0)}\right) & \geqslant \mathrm{E}_{x^{(0)}}\left[1_{\left\{\tau_{r} \leqslant T\right\}} \hat{u}(r)\right]+\mathrm{E}_{x^{(0)}}\left[1_{\left\{\tau_{r}>T\right\}} u(X(T))\right] \\
& =\hat{u}(r) P_{x^{0}}\left(A_{T}\right)+\mathrm{E}_{x^{(0)}}\left[1_{A_{T}^{\mathrm{c}}}^{\mathrm{c}} u\left(X_{T}\right)\right] \\
& >\hat{u}(r)(1-\varepsilon) .
\end{aligned}
$$

But this contradicts (ii) above. Hence (4.3) holds and step 1 is complete.

Step 2. We will show that, for all $x \in S_{1}$,

$$
P_{x}\left(\tau_{r}<\infty\right)=1 \text {. }
$$

Put

$$
\eta_{2 k}=\inf \left\{t>\eta_{2 k-1}: X_{1}(t)=1\right\} ; \quad \eta_{2 k+1}=\inf \left\{t>\eta_{2 k}: X_{1}(t)=2\right\} .
$$

Since the process $\left\{X_{1}(t)\right\}$ is recurrent note that $P_{x}\left(\eta_{k}<\infty\right)=1$ for all $k$. Hence by condition (iv), Itô's lemma and the optional sampling theorem we have

$$
\mathrm{E}_{x}\left[u\left(X\left(\eta_{k} \wedge \tau_{r}\right)\right)\right] \leqslant u(x),
$$

for all $k$. Let $A=\left\{\tau_{r}<\infty\right\}$ and $B=\left\{\eta_{k}<\infty\right.$ for all $\left.k\right\}$. Suppose (4.7) does not hold. Then $P_{x}\left(A^{\mathrm{c}}\right)>0$, and hence by recurrence of $\left\{X_{1}(t)\right\}$ we have $P_{x}\left(A^{\mathrm{c}} \cap B\right)>0$. By transience of the 
process (by step 1) and as $X_{1}$ and $X_{2}$ are bounded below by $r \wedge 0$ on $A^{\mathrm{c}}$, note that for a.a. $\omega \in A^{\mathrm{c}}$,

$$
X_{1}(t, \omega)+X_{2}(t, \omega) \rightarrow \infty \quad \text { as } t \rightarrow \infty .
$$

This implies that, for a.a. $\omega \in A^{\mathrm{c}} \cap B$,

$$
X_{2}\left(\eta_{k}(\omega), \omega\right) \rightarrow \infty \quad \text { as } k \rightarrow \infty .
$$

Now by (4.8) and condition (iii)

$$
u(x) \geqslant \lim _{k \rightarrow \infty} \mathrm{E}_{x}\left[u\left(X\left(\eta_{k} \wedge \tau_{r}\right)\right)\right] \geqslant \lim _{k \rightarrow \infty} \mathrm{E}_{x}\left[1_{A^{\mathrm{c}} \cap B} u\left(X\left(\tau_{r} \wedge \eta_{k}\right)\right)\right]=\infty,
$$

which is a contradiction. Hence (4.7) holds and step 2 is complete.

Step 3. Let $x=\left(x_{1}, x_{2}\right)$ be arbitrarily chosen. Choose $r, r_{1}$ such that $r_{1}<r<x_{2}$. Define

$$
\delta_{r_{1}}=\sup \left\{P_{y}\left(\tau_{r_{1}}<\infty\right): y \text { such that } y_{2}=r\right\} .
$$

By step $1, \delta_{r_{1}}<1$. By the strong Markov property,

$$
\begin{aligned}
P_{x}\left(X_{2}(t)\right. & \left.=r \text { for a sequence of } t^{\prime} \mathrm{s} \uparrow \infty\right) \leqslant P_{x}\left(\xi_{2 i+1}<\infty\right) \\
& =\mathrm{E}_{x}\left[1_{\zeta_{2 i-1<\infty}} P_{X\left(\xi_{2 i-1}\right)}\left(\tau_{r}<\infty\right)\right] \leqslant \delta_{r_{1}} P_{x}\left(\xi_{2 i-1}<\infty\right) \leqslant \ldots \leqslant \delta_{r_{i}}^{i},
\end{aligned}
$$

where

$$
\begin{gathered}
\xi_{2 i}=\inf \left\{t>\xi_{2 i-1}: X_{2}(t)=r\right\} \\
\zeta_{2 i+1}=\inf \left\{t>\zeta_{2 i}: X_{2}(t)=r_{1}\right\}, i=1,2, \ldots .
\end{gathered}
$$

As $\delta_{r_{1}}<1$ note that $\delta_{r_{1}}^{i} \rightarrow 0$. Hence $P_{x}\left(\limsup _{t \rightarrow \infty} X_{2}(t)<r\right)=1$. As $r<0$ is arbitrary, the proposition is proved.

Example. Consider the function $u(x)=\mathrm{e}^{x_{2}}$. Let $L, J$ be defined as in (4.1). Then $L u(x)=\left(a_{22}(x)+b_{2}(x)\right) \mathrm{e}^{x_{2}}$ and $J u(x)=\gamma_{2}\left(x_{2}\right) \mathrm{e}^{x_{2}}$. Hence, if $a_{22}(x)+b_{2}(x) \leqslant 0$ and $\gamma_{2}\left(x_{2}\right) \leqslant 0$, we have, on assuming the recurrence of $X_{1}$, that the process is transient down to $-\infty$.

Note. Conditions for recurrence of $X_{1}$ are being investigated. In this connection one may consult Ramasubramanian (1983) for conditions for recurrence of projections of diffusions in $\mathbb{R}^{d}$ (without boundary conditions). Such conditions (together with appropriate modifications required to ensure that the derivatives along the reflecting directions are negative) in the present context are not difficult to prove.

\section{Positive recurrence of diffusions in the half-space}

In this section we will deal with positive recurrence of diffusions and the existence of invariant measures. First, let us define some stopping times which will be used below. We consider diffusions in the half-space $\bar{D}$, where

$$
D=\left\{\left(x_{1}, x_{2}, \ldots, x_{d}\right): x_{1}>0\right\} .
$$


Let $U$ be an open set in $\bar{D}$. Define

$$
\begin{aligned}
\tau_{U} & =\inf \{t \geqslant 0: X(t) \notin U\}=\text { exit time from } U . \\
\sigma_{U} & =\inf \{t \geqslant 0: X(t) \in U\}=\text { entrance time into } U .
\end{aligned}
$$

For $c>0$, define

$$
\tau_{c}=\inf \{t \geqslant 0:|X(t)|=c\}
$$

Definition. A point $x \in \bar{D}$ is said to be positive recurrent if there exist bounded open sets $U_{1}$, $U_{2}$ such that $x \in U_{1} \subseteq \bar{U}_{1} \subseteq U_{2}$ and

$$
\sup \left\{\mathrm{E}_{z}\left(\sigma_{U_{1}}\right): z \in \partial U_{2}\right\}<\infty .
$$

The diffusion is said to be positive recurrent if all points are recurrent.

Lemma 5.1. Let $x$ be a positive recurrent point; let $U_{1}, U_{2}$ be open balls such that (5.1) holds. Let $U, V$ be balls such that $\bar{U}_{1} \subseteq U \subseteq \bar{U} \subseteq U_{2} \subseteq \bar{U}_{2} \subseteq V$. Then

$$
\begin{aligned}
& \sup \left\{\mathrm{E}_{z}\left(\sigma_{U_{1}}\right): z \in \partial U\right\}<\infty \\
& \sup \left\{\mathrm{E}_{z}\left(\sigma_{U_{1}}\right): z \in \partial V\right\}<\infty .
\end{aligned}
$$

Proof. By the strong Markov property, Lemma 3 of Ramasubramanian (1986) and by positive recurrence of $x$, we have

$$
\begin{aligned}
\sup _{y \in \partial U} \mathrm{E}_{y}\left(\sigma_{U_{1}}\right) & \leqslant \sup _{y \in \partial U} \mathrm{E}_{y}\left[1_{\sigma_{U_{1}}}<\tau_{U_{2}} \cdot \sigma_{U_{1}}\right]+\sup _{y \in \partial U} \mathrm{E}_{y}\left[1_{\sigma_{U_{1}}>\tau_{U_{2}}} \cdot \sigma_{U_{1}}\right] \\
& \leqslant \sup _{y \in \partial U} \mathrm{E}_{y}\left(\tau_{U_{2}}\right)+\sup _{y \in \partial U} \mathrm{E}_{y}\left[\mathrm{E}_{X\left(\tau_{U_{2}}\right)}\left(\sigma_{U_{1}}\right)\right] \\
& <\infty .
\end{aligned}
$$

By Proposition 2.3, the existence of a positive recurrent point implies that the diffusion is recurrent and hence we have $\sigma_{U_{2}}<\infty, P_{z}$ a.s. for $z \in \bar{U}_{2}^{\mathrm{c}}$. Therefore we have

$$
\begin{aligned}
\sup _{z \in \partial V} \mathrm{E}_{z}\left(\sigma_{U_{1}}\right) & =\sup _{z \in \partial V} \mathrm{E}_{z}\left[\mathrm{E}_{z}\left(\sigma_{U_{1}} \mid \mathscr{B}_{\sigma_{U_{2}}}\right)\right] \\
& =\sup _{z \in \partial V} \mathrm{E}_{z}\left(\mathrm{E}_{X\left(\sigma_{U_{2}}\right)}\left(\sigma_{U_{1}}\right)\right. \\
& \leqslant \sup _{z \in \partial U_{2}} \mathrm{E}_{z}\left(\sigma_{U_{1}}\right)<\infty .
\end{aligned}
$$

Proposition 5.2. If there exists one positive recurrent point, then the diffusion itself is positive recurrent.

Proof. Let $x_{0}$ be a positive recurrent point and let $y$ be an arbitrary point. We will show that $y$ is positive recurrent. Since $x_{0}$ is a positive recurrent point, we can find two balls $U_{1}, U_{2}$ 
such that (5.1) holds. Let $U_{3}, U_{4}$ be balls such that $\bar{U}_{2} \subseteq U_{3} \subseteq \bar{U}_{3} \subseteq U_{4}$ and $y \in U_{3}$. By Lemma 5.1,

$$
\sup \mathrm{E}_{z}\left\{\sigma_{U_{1}}: z \in \partial U_{4}\right\}<\infty .
$$

Since $\sigma_{U_{3}}<\sigma_{U_{1}}, P_{z}$ a.s. for $z \in \partial U_{4}$, we have

$$
\sup \left\{\mathrm{E}_{z}\left(\sigma_{U_{3}}: z \in \partial U_{4}\right\}<\infty .\right.
$$

Combining (5.6) and (5.7), we see that $y$ is a positive recurrent point. As $y$ was chosen to be arbitrary, the diffusion is positive recurrent.

Our next objective is to obtain an upper bound for the expected hitting time of a bounded open set. For this we need the following lemma.

Lemma 5.3. Let $A$ be a bounded open set in $\bar{D}$, and let $r>0$ be such that $\bar{A} \subseteq B(0: r)$. Then there exist $M>r, 0<p_{A}<1$, such that, for all $x \in \overline{B(0: r)}$,

$$
P_{x}(X(1) \in A \text { and }|X(t)| \leqslant M, \forall t \in[0,1]) \geqslant p_{A} .
$$

Proof. Since the diffusion has a continuous positive density and $A$ is an open set, note that

$$
p_{0} \equiv \inf \left\{P_{x}(X(1) \in A): x \in \overline{B(0: r)}\right\}>0 .
$$

Let $\varepsilon=p_{0} / 4$. By tightness of measures $\left\{P_{x}: x \in \overline{B(0: r)}\right\}$ on $C\left([0,1]: \mathbb{R}^{d}\right)$, we can find a compact set $K_{\varepsilon} \subseteq C\left([0,1]: \mathbb{R}^{d}\right)$ such that, for all $x \in \overline{B(0: r)}$,

$$
P_{x}\left(K_{\varepsilon}\right)>1-\varepsilon .
$$

By Arzela-Ascoli's theorem, there exists $M>0$ such that $|\omega(t)| \leqslant M$, for all $t \in[0,1]$, for all $\omega \in K_{\varepsilon}$. Hence, for all $x \in \overline{B(0: r)}$, by (5.9) we have

$$
\begin{aligned}
P_{x}\left(X_{1}^{-1}(A) \cap K_{\varepsilon}\right) & =P_{x}\left(X_{1}^{-1}(A)\right)-P_{x}\left(X_{1}^{-1}(A) \backslash K_{\varepsilon}\right) \\
& >p_{0}-\varepsilon=\frac{3}{4} p_{0}=: p_{A}
\end{aligned}
$$

whence the lemma follows.

Now with $M, r$ as in the preceding lemma, let $\eta_{0}=0$ and put:

$$
\begin{aligned}
\eta_{1} & =\inf \{t \geqslant 0:|X(t)|=r\} \vee\left(1 \wedge \tau_{M}\right) ; \\
\eta_{2 i} & =\inf \left\{t>\eta_{2 i-1}: X(t) \notin B(0: M)\right\} \wedge\left(\eta_{2 i-1}+1\right) ; \\
\eta_{2 i+1} & =\inf \left\{t>\eta_{2 i}: X(t) \in \partial B(0: r)\right\} .
\end{aligned}
$$

Let $F=\left\{\sigma_{A} \leqslant 1, \tau_{M}>1\right\}$, where $A, M$ are as in the preceding lemma. By (5.10) note that

$$
P_{x}(F)>p_{A} \text {. }
$$

Proposition 5.4. Let $A, r, M, p_{A}$ be as in Lemma 5.3. For any $x \in \overline{B(0: r)}$, 


$$
\mathrm{E}_{x}\left(\sigma_{A}\right) \leqslant \frac{1}{p_{A}}\left[2+\sup _{|z| \leqslant M} \mathrm{E}_{z}\left(\tau_{r}\right)\right]+\mathrm{E}_{x}\left(\tau_{r}\right)
$$

Proof. If $\sup _{|z| \leqslant M} \mathrm{E}_{z}\left(\tau_{r}\right)=\infty$, note that (5.12) trivially holds. So assume $\sup _{|z| \leqslant M} \mathrm{E}_{z}\left(\tau_{r}\right)<\infty$. Then note that any point in $B(0: r)$ is positive recurrent and hence the diffusion itself is positive recurrent. This, in particular, implies that the diffusion is recurrent. Therefore all the stopping times involved in the proof are well defined.

$$
\begin{aligned}
\mathrm{E}_{x}\left(\sigma_{A}\right)= & \mathrm{E}_{x}\left[\int_{0}^{\sigma_{A} \wedge \eta_{1}} \mathrm{~d} s\right]+\mathrm{E}_{x}\left[\sum_{i \geqslant 1} \int_{\sigma_{A} \wedge \eta_{2 i-1}}^{\sigma_{A} \wedge \eta_{2 i}} \mathrm{~d} s\right] \\
& +\mathrm{E}_{x}\left[\sum_{i \geqslant 1} \int_{\sigma_{A} \wedge \eta_{2 i}}^{\sigma_{A} \wedge \eta_{2 i+1}} \mathrm{~d} s\right] .
\end{aligned}
$$

Clearly,

$$
\begin{aligned}
\mathrm{E}_{x}\left[\int_{0}^{\sigma_{A} \wedge \eta_{1}} \mathrm{~d} s\right] \leqslant \mathrm{E}_{x}\left(\eta_{1}\right) & =\mathrm{E}_{x}\left(\tau_{r} \vee\left(1 \wedge \tau_{M}\right)\right) \\
& \leqslant 1+\mathrm{E}_{x}\left(\tau_{r}\right) .
\end{aligned}
$$

Next, by the strong Markov property for $i \geqslant 1$,

$$
\begin{aligned}
\mathrm{E}_{x}\left[\int_{\sigma_{A} \wedge \eta_{2 i-1}}^{\sigma_{A} \wedge \eta_{2 i}} \mathrm{~d} s\right]= & \mathrm{E}_{x}\left[1_{\left\{\sigma_{A}>\eta_{2 i-1}\right\}} \mathrm{E}_{X\left(\eta_{2 i-1}\right)} \int_{0}^{\sigma_{A} \wedge \tau_{M} \wedge 1} \mathrm{~d} s\right] \\
& \leqslant P_{x}\left(\sigma_{A}>\eta_{2 i-1}\right) .
\end{aligned}
$$

As $\left\{\sigma_{A}>\eta_{1}\right\} \subseteq F^{\mathrm{c}}$, we have

$$
P_{x}\left(\sigma_{A}>\eta_{1}\right) \leqslant\left(1-p_{A}\right)
$$

Now observe that, for $i \geqslant 3$,

$$
\begin{aligned}
P_{x}\left(\sigma_{A}>\eta_{2 i-1}\right)= & \mathrm{E}_{x}\left[1_{\left\{\sigma_{A}>\eta_{2 i-1}\right\}} 1_{\left\{\sigma_{A}>\eta_{2 i-3}\right\}}\right] \\
& \leqslant \mathrm{E}_{x}\left[1_{\left\{\sigma_{A}>\eta_{2 i-3}\right\}} \mathrm{E}_{X\left(\eta_{2 i-3}\right)}\left(1_{\left\{\sigma_{A}>\left(\tau_{M} \wedge 1\right)\right\}}\right)\right] \\
& \leqslant P_{x}\left(\sigma_{A}>\eta_{2 i-3}\right) \sup _{|z| \leqslant r} P_{z}\left(\sigma_{A}>\left(\tau_{M} \wedge 1\right)\right) \\
& \leqslant\left(1-p_{A}\right) P_{x}\left(\sigma_{A}>\eta_{2 i-3}\right) .
\end{aligned}
$$

As $\eta_{3}>\eta_{1}$ a.s., by (5.16) we have

$$
P_{x}\left(\sigma_{A}>\eta_{3}\right) \leqslant\left(1-p_{A}\right) .
$$

By (5.17) and (5.18) we have

$$
P_{x}\left(\sigma_{A}>\eta_{2 i-1}\right) \leqslant\left(1-p_{A}\right)^{i-1} .
$$


Combining (5.15), (5.16) and (5.19), we have

$$
\mathrm{E}_{x}\left[\sum_{i \geqslant 1} \int_{\sigma_{A} \wedge \eta_{2 i-1}}^{\sigma_{A} \wedge \eta_{2 i}} \mathrm{~d} s\right] \leqslant \frac{1}{p_{A}}+\left(1-p_{A}\right) .
$$

By the strong Markov property and the fact that $X\left(\eta_{2 i}\right) \in \overline{B(0: M)}$, we have

As $\eta_{2 i}>\eta_{2 i-1}$ a.s., we have

$$
\begin{aligned}
\mathrm{E}_{x}\left[\int_{\sigma_{A} \wedge \eta_{2 i}}^{\sigma_{A} \wedge \eta_{2 i+1}} \mathrm{~d} s\right]= & \mathrm{E}_{x}\left[1_{\left\{\sigma_{A}>\eta_{2 i}\right\}} \mathrm{E}_{X\left(\eta_{2 i}\right)} \int_{0}^{\sigma_{A} \wedge \tau_{r}} \mathrm{~d} s\right] \\
& \leqslant P_{x}\left(\sigma_{A}>\eta_{2 i}\right) \sup _{|z| \leqslant M} \mathrm{E}_{z}\left(\tau_{r}\right) .
\end{aligned}
$$

$$
P_{x}\left(\sigma_{A}>\eta_{2 i}\right) \leqslant P_{x}\left(\sigma_{A}>\eta_{2 i-1}\right) \leqslant\left(1-p_{A}\right)^{i-1} .
$$

Combining (5.21) and (5.22), we obtain

$$
\mathrm{E}_{x}\left[\sum_{i \geqslant 1} \int_{\sigma_{A} \wedge \eta_{2 i}}^{\sigma_{A} \wedge \eta_{2 i+1}} \mathrm{~d} s\right] \leqslant \frac{1}{p_{A}}\left[\sup _{|z| \leqslant M} \mathrm{E}_{z}\left(\tau_{r}\right)\right] .
$$

Now combining equations (5.13), (5.14), (5.20) and (5.23), we have the proposition.

Corollary 5.5. If the diffusion is positive recurrent then $\mathrm{E}_{y}\left(\sigma_{A}\right)<\infty$, for any $y \in \bar{D}$ and for any non-empty open set $A \subset \bar{D}$.

Proof. Without loss of generality take $A$ to be bounded open. Let $y \in \bar{D}$ be arbitrary but fixed. Let $x \in A$ be arbitrary. By positive recurrence there exist open balls $U_{1}, U_{2}$ such that $x \in U_{1} \subseteq \bar{U}_{1} \subseteq U_{2}$ and (5.1) holds. Now choose $r>|y|$ such that $\bar{A} \cup \bar{U}_{1} \subseteq B(0: r)$. For any $M$ such that $\overline{B(0: r)} \cup \bar{U}_{2} \subset B(0: M), \sup _{|z| \leqslant M} \mathrm{E}_{z}\left(\sigma_{U_{1}}\right)<\infty$ by Lemma 5.1. Hence we have $\sup _{|z| \leqslant M} \mathrm{E}_{z}\left(\tau_{r}\right)<\infty$, by continuity of sample paths. Choose a suitable $M$, such that Lemma 5.3 and Proposition 5.4 hold. Now the corollary follows.

Proposition 5.6. Let $r_{0}>0, \varepsilon>0, u \in C^{2}\left(\mathbb{R}^{d} \backslash B\left(0: r_{0} / 2\right)\right)$ be such that

(i) $L u(x) \leqslant-\varepsilon,|x| \geqslant r_{0}$;

(ii) $J u(x) \leqslant 0,\left\{|x| \geqslant r_{0}\right\} \cap \partial D$;

(iii) $u(x) \geqslant 0$ for all $x$ such that $|x| \geqslant r_{0}$.

Then the diffusion is positive recurrent.

Proof. Let $\tau_{r_{0}}^{n}=\inf \left\{t \geqslant 0:|X(t)| \notin\left(r_{0}, n\right)\right\}$. Then by Itô's formula,

$$
\mathrm{E}_{x}\left[u\left(X\left(t \wedge \tau_{r_{0}}^{n}\right)\right)\right]-u(x) \leqslant-\varepsilon \mathrm{E}_{x}\left(t \wedge \tau_{r_{0}}^{n}\right) .
$$

So $\mathrm{E}_{x}\left(t \wedge \tau_{r_{0}}^{n}\right) \leqslant\left[u(x)-\mathrm{E}_{x}\left(u\left(X\left(t \wedge \tau_{r_{0}}^{n}\right)\right)\right)\right] / \varepsilon$. Hence $\mathrm{E}_{x}\left(\tau_{r_{0}}^{n}\right) \leqslant u(x) / \varepsilon$ as $t \rightarrow \infty$. But since $\tau_{r_{0}}^{n} \uparrow \tau_{r_{0}}$ as $n \rightarrow \infty$, we have 


$$
\mathrm{E}_{x}\left(\tau_{r_{0}}\right) \leqslant \frac{u(x)}{\varepsilon}<\infty .
$$

From (5.25) it follows that $\sup _{|z|=r} \mathrm{E}_{z}\left(\tau_{r_{0}}\right)<\infty$ for any $r>r_{0}$. Hence we have that the process is positive recurrent.

Remark 5.1. If the diffusion is recurrent, then, by the argument and results of Maruyama and Tanaka (1959), there exists a unique (up to scalar multiplicity) $\sigma$-finite invariant measure. Furthermore, if the diffusion is positive recurrent, then, by the same arguments, the invariant measure is a probability measure. (Note that the Condition 6 of Maruyama and Tanaka (1959) is needed just for open balls.)

Remark 5.2. In Bhattacharya (1978) a point $x$ is said to be positive recurrent if, for all $0<r_{0}<r_{1}$, we have $\mathrm{E}_{z}\left(\sigma_{B\left(x: r_{0}\right)}\right)<\infty$, for all $z \in \partial B\left(x: r_{1}\right)$. However, to prove the existence of an invariant probability measure a condition similar to (5.1) above is needed.

Remark 5.3. Estimate (5.12) is stated (with a brief indication of proof) in Dupuis and Williams (1994), in the context of semimartingale RBMs in the orthant. As this estimate is likely to be very useful we thought it appropriate to write up a proof.

Note. Our analysis concerning recurrence, transience and positive recurrence can easily be extended to unbounded domains that are $C^{2}$-diffeomorphic to the half-space.

\section{Further comments and examples}

Example 6.1. Let $D=\left\{\left(x_{1}, x_{2}\right): x_{1}>0\right\}$. Put $L=\Delta / 2-\mu \partial / \partial x_{1}, \mu>0$. Note that in $\mathbb{R}^{2}$, $L$-diffusion is transient as the diffusion is $\left(B_{1}(t)-\mu t, B_{2}(t)\right)$, where $B_{1}$ and $B_{2}$ are Brownian motions.

But let us consider $(L, J)$-diffusion where $J=\partial / \partial x_{1}$. Now by taking $u(x)=\log |x|$, we see that

$$
\begin{array}{ll}
L u \leqslant 0 & \text { on } D ; \\
J u \leqslant 0 & \text { on } \partial D .
\end{array}
$$

Furthermore, $u(x) \rightarrow \infty$ as $|x| \uparrow \infty$. Hence the process is recurrent, by Corollary 2.5 .

Example 6.2. Now consider

$$
D=\left\{\left(x_{1}, x_{2}\right): x_{2}>0\right\} .
$$

Let $\beta_{1}, \beta_{2}$ be negative constants, and

$$
\begin{aligned}
& L f(x)=\Delta f(x)+\beta_{1} x_{1} \frac{\partial f(x)}{\partial x_{1}}+\beta_{2} x_{2} \frac{\partial f(x)}{\partial x_{2}} \\
& J f(x)=\gamma_{1}\left(x_{1}\right) \frac{\partial f(x)}{\partial x_{1}}+\frac{\partial f(x)}{\partial x_{2}} ;
\end{aligned}
$$


that, is we consider an Ornstein-Uhlenbeck process in the upper half-plane with reflection field $\left(\gamma_{1}\left(x_{1}\right), 1\right)$. We can have positive recurrence of the process in the following cases.

Case (i). Let $\gamma_{1}\left(x_{1}\right) \leqslant 0$, for $x_{1} \geqslant 1$, and $\gamma_{1}\left(x_{1}\right) \geqslant 0$, for $x_{1} \leqslant-1$. Then with the function $f(x)=\log |x|$, applying Proposition 5.6, we can see that the process is positive recurrent.

Case (ii). Let $\beta_{1}=\beta_{2}=\beta$. Now consider the upper half-plane in polar coordinate form, that is,

$$
D=\{(r, \theta): r>0, \theta \in(0, \pi)\} .
$$

Then the diffusion and boundary operators transform to

$$
\begin{gathered}
L_{r, \theta}=\frac{\partial}{\partial r^{2}}+\frac{1}{r} \frac{\partial}{\partial r}+\frac{1}{r^{2}} \frac{\partial}{\partial \theta^{2}}+\beta r \frac{\partial}{\partial r} \\
J= \begin{cases}\tan \eta(\cdot) \frac{\partial}{\partial r}+\frac{1}{r} \frac{\partial}{\partial \theta} & \text { on } \theta=0 \\
-\tan \eta(\cdot) \frac{\partial}{\partial r}-\frac{1}{r} \frac{\partial}{\partial \theta} & \text { on } \theta=\pi .\end{cases}
\end{gathered}
$$

Now if the reflection field satisfies the condition in Theorem 3.1(a), then by Proposition 5.6 applied to the function $u(r, \theta)$ as in the corresponding proof, we have positive recurrence of the Ornstein-Uhlenbeck process.

Similarly, if the reflection field satisfies the condition in Theorem 3.1(c), we have positive recurrence of the Ornstein-Uhlenbeck process. In particular, we see that the OrnsteinUhlenbeck process with constant angles of reflection is positive recurrent.

Example 6.3. Let $S$ be the upper half-plane as in Section 3. Let

$$
L f(x)=\sum_{i, j=1}^{2} a_{i j}(x) \frac{\partial^{2} f(x)}{\partial x_{i} \partial x_{j}},
$$

where

$$
a_{i j}(x)=\delta_{i j}+\frac{g(r)}{r^{2}} x_{i} x_{j},
$$

in which $g(r)$ is a bounded Lipschitz continuous function. Note that $S=\{(r, \theta): r>0, \theta \in$ $(0, \pi)\}$. Let $J$ be given by (3.1). In polar coordinates $L$ above is transformed to

$$
L=(1+g(r)) \frac{\partial^{2}}{\partial r^{2}}+\frac{1}{r} \frac{\partial}{\partial r}+\frac{1}{r^{2}} \frac{\partial^{2}}{\partial \theta^{2}} .
$$

Case (i). Let the reflection field satisfy the condition in Theorem 3.1(a). Assume $g(r) \geqslant 0$. Take $\alpha, s_{0}$ and $u$ as in the proof of Theorem 3.1(a). Note that $0<\alpha<1$. Then on $\left[\partial_{1} S \cap\left\{r \geqslant s_{0}\right\}\right] \cup\left[\partial_{2} S \cap\left\{r \geqslant s_{0}\right\}\right]$ we have

$$
J u \leqslant 0 \text {. }
$$

As $g(r) \geqslant 0$, we have

$$
L u \leqslant g(r) \alpha(\alpha-1) r^{\alpha-2} \cos \left(\alpha \theta-\theta_{1}\right) \leqslant 0,
$$


and in this case the process will be recurrent.

Case (ii). Let the reflection field satisfy the condition in Theorem 3.1(b). Assume $g(r)<0$. Take $\alpha, u$ as in the proof of Theorem 3.1(b). Since $\alpha<0$ we choose $s_{0}>0$ such that, on $\left[\partial_{1} S \cap\left\{r \geqslant s_{0}\right\}\right] \cup\left[\partial_{2} S \cap\left\{r \geqslant s_{0}\right\}\right]$, we have

$$
J u \leqslant 0 \text {. }
$$

As $\alpha<0$ and $g(r)<0$, note that

$$
L u \leqslant 0 .
$$

Hence the process is transient. In particular, if $g(r)=-1 /(1+\log r)$, we have recurrence in the unrestricted case (see Friedman 1975, p. 202). But in the upper half-plane with the reflection as above, the process is transient.

Example 6.4. Let $\left.D=\left(x_{1}, x_{2}, x_{3}, x_{4}\right): x_{1}>0\right\}$ and let $\left(1, \gamma_{2}, \gamma_{3}, \gamma_{4}\right)$ be the reflection field on $\partial D$, where $\gamma_{2}, \gamma_{3}$ and $\gamma_{4}$ are constants. Consider Brownian motion in $\bar{D}$ with reflection field as above. The equation can be explicitly written for reflection Brownian motion in $\bar{D}$,

$$
\begin{aligned}
& Z_{1}(t)=B_{1}(t)+\xi(t) \\
& Z_{2}(t)=B_{2}(t)+\gamma_{2} \xi(t) \\
& Z_{3}(t)=B_{3}(t)+\gamma_{3} \xi(t) \\
& Z_{4}(t)=B_{4}(t)+\gamma_{4} \xi(t)
\end{aligned}
$$

where $\xi(t)$ is the local time at 0 for the Brownian motion $B_{1}(t)$, and the Brownian motions $B_{1}(t), B_{2}(t), B_{3}(t)$ and $B_{4}(t)$ are independent. Without loss of generality, assume that $\gamma_{2}^{2}+\gamma_{3}^{2}+\gamma_{4}^{2}=1$. Let $O$ be the orthogonal transformation in $\mathbb{R}^{3}(\cong \partial D)$ taking $\left(\gamma_{2}, \gamma_{3}, \gamma_{4}\right)$ to $(1,0,0)$. Hence

$$
\begin{aligned}
O\left(Z_{2}, Z_{3}, Z_{4}\right)^{\mathrm{T}} & =O\left(B_{2}, B_{3}, B_{4}\right)^{\mathrm{T}}+O\left(\gamma_{2}, \gamma_{3}, \gamma_{4}\right)^{\mathrm{T}} \xi(t) \\
& =\left(\tilde{B}_{2}, \tilde{B}_{3}, \tilde{B}_{4}\right)^{\mathrm{T}}+(\xi(t), 0,0)^{\mathrm{T}}
\end{aligned}
$$

where $\left(\tilde{B}_{2}, \tilde{B}_{3}, \tilde{B}_{4}\right)$ is again a three-dimensional Brownian motion. Consider the transformation $T: \mathbb{R}^{4} \rightarrow \mathbb{R}^{4}$ such that $T\left(x_{1}, x_{2}, x_{3}, x_{4}\right)=\left(x_{1}, y_{2}, y_{3}, y_{4}\right)$, where $\left(y_{2}, y_{3}, y_{4}\right)^{\mathrm{T}}=$ $O\left(x_{2}, x_{3}, x_{4}\right)^{\mathrm{T}}$. As $T$ is a smooth transformation, it would preserve recurrence and transience. Let $T\left(Z_{1}, Z_{2}, Z_{3}, Z_{4}\right)=\left(\tilde{Z}_{1}, \tilde{Z}_{2}, \tilde{Z}_{3}, \tilde{Z}_{4}\right)$, where

$$
\begin{aligned}
& \tilde{Z}_{1}(t)=B_{1}(t)+\xi(t) \\
& \tilde{Z}_{2}(t)=\tilde{B}_{2}(t)+\xi(t) \\
& \tilde{Z}_{3}(t)=\tilde{B}_{3}(t) \\
& \tilde{Z}_{4}(t)=\tilde{B}_{4}(t) .
\end{aligned}
$$

Now note that $\left(\tilde{Z}_{1}, \tilde{Z}_{3}, \tilde{Z}_{4}\right)$ is a three-dimensional reflected Brownian motion with normal reflection in the space $\bar{E}=\left\{\left(x_{1}, x_{3}, x_{4}\right): x_{1} \geqslant 0\right\}$ and is transient. Hence the diffusion $\left(\tilde{Z}_{1}, \tilde{Z}_{2}, \tilde{Z}_{3}, \tilde{Z}_{4}\right)$ is transient. In general this result is true for dimensions greater than 4 . 
To conclude, we mention two cases in which the asymptotic behaviour of the diffusion is not clear to the authors.

(a) Brownian motion in three dimensions with reflection field $\gamma(x)$, where $\gamma$ is bounded smooth. One would expect this process to be transient; however, even when $\gamma \equiv$ constant we do not know the result.

(b) For the Ornstein-Uhlenbeck process in the half-plane with drift coefficients $\beta_{1} x_{1}, \beta_{2} x_{2},\left(\beta_{1}<0, \beta_{2}<0\right)$ we do not know whether the process is recurrent. In particular, we do not know the behaviour of the process when $\gamma$ is such that $\langle x, \gamma(x)\rangle>0$, for all $x \in \partial D$. Also we are not able to say anything about positive recurrence.

\section{Acknowledgement}

The authors express their gratitude to three painstaking referees for their encouraging comments and suggestions. Remark 2.9 arose out of comment by one referee. Another referee brought to our notice the paper of Menshikov and Williams (1995) and the possibility of establishing Proposition 3.5. We also thank all three referees for pointing out several misprints. Thanks are also due to Prof. R. J. Williams for promptly sending us the preprint of Menshikov and Williams (1996).

\section{References}

Bhattacharya, R.N. (1978) Criteria for recurrence and existence of invariant measures for multidimensional diffusions. Ann. Probab., 6, 541-553.

Bhattacharya, R.N. and Waymire, E.C. (1990) Stochastic Processes with Applications. New York: Wiley.

Dupuis, P. and Williams, R.J. (1994) Lyapunov functions for semimartingale reflecting Brownian motions. Ann. Probab. 22, 680-702.

Dynkin, E.B. (1965) Markov Processes, Vol. II. New York: Springer-Verlag.

Friedman, A. (1975) Stochastic Differential Equations and Applications, Vol. 1, New York: Academic Press.

Ichihara, K., (1978) Some global properties of symmetric diffusion processes. Publ. Res. Inst. Math. Sci. 14, 441-486.

Khasminskii, R.Z. (1960) Ergodic properties of recurrent diffusion processes and stabilization of the solution of the Cauchy problem for parabolic equations. Theory Probab. Appl., 5, 179-196.

Maruyama, G. and Tanaka, H. (1959) Ergodic property of $N$-dimensional recurrent Markov processes. Mem. Fac. Sci. Kyushu Univ. Ser. A, 13, 157-172.

Menshikov, M. and Williams, R.J. (1996) Passage-time moments for continuous nonnegative stochastic processes and applications. Adv. Appl. Prob., 28, 747-762.

Meyn, S.P. and Tweedie, R.L. (1993a) Stability of Markovian processes II: Continuous time processes and sampled chains. Adv. Appl. Probab., 25, 487-517.

Meyn, S.P. and Tweedie, R.L. (1993b) Stability of Markovian processes III: Foster-Lyapunov criteria for continuous time processes. Adv. Appl. Probab., 25, 518-548. 
Pinsky, R.G. (1987) Recurrence, transience and bounded harmonic functions for diffusions in the plane. Ann. Probab., 15, 954-984.

Pinsky, R.G. (1995) Positive Harmonic Functions and Diffusion. Cambridge: Cambridge University Press.

Protter, M.H. and Weinberger, H.F. (1967) Maximum Principles in Differential Equations. Englewood Cliffs, NJ: Prentice Hall.

Ramasubramanian, S. (1983) Recurrence of projections of diffusions. Sankhya Ser. A, 45, 20-31.

Ramasubramanian, S. (1986) Hitting a boundary point by diffusions in closed half space. J. Multivariate Anal., 20, 143-154.

Ramasubramanian, S. (1988) Hitting of submanifolds by diffusions. Probab. Theory Related Fields, 78, $149-163$.

Ramasubramanian, S. (1996) Transition densities of reflecting diffusions. Sankhyā Ser A, 58, 347-381.

Rogers L.C.G. (1990) Brownian motion in a wedge with variable skew reflection II. In M. Pinsky (ed) Diffusion Processes and Related Problems in Analysis, pp. 95-115. Boston: Birkhäuser.

Rogers, L.C.G. (1991) Brownian motion in a wedge with variable skew reflection. Trans. Amer. Math. Soc., 326, 221-236.

Stroock, D.W. and Varadhan, S.R.S. (1971) Diffusion processes with boundary conditions. Comm. Pure Appl. Math. 24, 147-225.

Stroock, D.W. and Varadhan, S.R.S (1972) On the support of diffusion processes with applications to strong maximum principle. In L. Le Cam and J. Neyman (eds) Proceedings of the Sixth Berkeley Symposium on Mathematical Statistics and Probability, Vol. 3, pp. 333-359. Berkeley: University of California Press.

Varadhan, S.R.S and Williams, R.J. (1985) Brownian motion in a wedge with oblique reflection. Comm. Pure Appl. Math., 38, 405-443.

Williams, R.J. (1985) Recurrence classification and invariant measure for reflecting Brownian motion in a wedge. Ann. Probab., 13, 758-778.

Received August 1995 and revised May 1996 